\title{
Digital Image Watermarking using New Combined Technique
}

\author{
Maninder Kaur \\ Department of Computer Engineering, \\ Punjabi University, Patiala, \\ Punjab
}

\author{
Nirvair Neeru \\ Department of Computer Engineering, \\ Punjabi University, Patiala, \\ Punjab
}

\begin{abstract}
In this paper a new method of digital image watermarking is proposed for the protection of digital contents. This proposed method presents a technique which is based on combination of spatial domain technique and frequency domain techniques. Discrete wavelet transform, singular value decomposition and least significant bit techniques are combined to provide robustness to the watermark image as well as to improve the quality of obtained watermarked image.
\end{abstract}

\section{General Terms}

Gray scale image, LSB, DWT, SVD, PSNR, MSE, SSIM.

\section{Keywords}

Keywords are your own designated keywords which can be used for easy location of the manuscript using any search engines.

\section{INTRODUCTION}

In today's world digital contents of different types like text, image, video and audio can be easily shared between two or more places with the use of internet. It provides a big advantage to the users of internet but it also has some problems. When any user sends digital content through internet there may be chances that any intruder can copy the digital content and use it without any knowledge to the original owner of data and the intruder can also claims that the digital content belongs to him. In this case a threat to original owner's right arise, also intruder can make modifications in the original digital content send by sender and forward it to receiver. Receiver cannot know that digital content received by him is not original. Thus to protect digital contents transferred over internet various means are used such as cryptography, digital watermarking and steganograpy. Digital watermarking is a method of inserting or embedding a secret data (also known watermark or label or tag or digital signature) in the original digital content with help of embedding algorithm. The original content in which watermark is embedded is called host. Watermark is embedded in such a way that the changes made in host are unnoticeable [1]. Types of watermarks used in insertion are visible watermark, invisible watermark, robust watermark and fragile watermark. Visible watermark is a text or opaque image which is embedded in a host digital image and it can be easily seen to human eyes. Whereas in case of invisible watermark no one is able to identify watermark embedded in a host image directly by having a look at the image [3]. Robust watermark are difficult to remove or modify or destroy by any type of attack like cropping, scaling or translation or addition of noise in watermarked image. Robust watermark provides security to the digital contents and they also help in identifying the original owner of digital contents. Whereas fragile watermark can be easily removed or destroyed or modified by any attack. Thus they are used in authentication of digital contents [2]. Digital watermarking techniques are categorized in spatial domain and frequency domain. In spatial domain changes are made directly in image pixel values whereas in frequency domain watermark is embedded in spectral coefficient of a host image.

Remaining part of paper is organized as following: related work is in section 2, proposed method is explained in section 3 , section 4 contains results of proposed method and conclusion is presented in section 5 .

\section{RELATED WORK}

Saeid Fazli et al. [5] proposed a robust watermarking method to reduce effect of geometric attacks on images. In this proposed method a technique for correction of geometric distortion of an image is also purposed. In this technique discrete wavelet transform is used to divide image into sub bands and then discrete cosine transform and singular value transform is used to embed watermark in $\mathrm{LH}$ and HL sub band of host image.

Hanaa A. Abdullah et al. [4] proposed a homomorphic image watermarking implemented using singular value decomposition (SVD) algorithm. As it is block based watermarking method so input image is sub divided into blocks of size $8 * 8$. In this approach high pass filters and low pass filters are used to extract reflectance component and illumination components of an input image and after that watermark is embedded in sub blocks is done by using singular value decomposition approach.

Samira Lagzaian et al. [6] proposed a robust watermarking scheme image watermarking using RDWT and SVD techniques of watermarking. In this proposed technique redundant wavelet transform and singular value decomposition techniques are applied to extract coefficients of host image and watermark image and then redundant wavelet transform is used to embed watermark in a host image. In this RDWT is used because it overcomes the disadvantages of discrete wavelet transform.

V. Santhi et al. [7] proposed discrete wavelet transform and singular value decomposition based technique for YUV and RGB colored images. In this proposed method first colored image is converted into YUV color components and after that discrete wavelet transform and singular value decomposition techniques of watermarking are used to insert watermark in an original image. On completion of watermarking YUV color components are again converted into original colored image.

\section{PROPOSED METHOD}

This digital watermarking method is proposed to provide security to images and also to improve the quality of obtained watermarked image. The proposed method is combination of three existing digital watermarking techniques that is combination of frequency domain based discrete wavelet 
transform technique (DWT), singular value decomposition (SVD) and spatial domain based least significant bit (LSB) technique applied on grey scale images. Least significant bit technique is used to improve the quality of obtained watermark image as in this technique modification is performed on least significant bit position which cannot be recognized by human eyes. Discrete wavelet transforms and singular value decomposition is applied to provide robustness to method. DWT technique decomposes an image into multiresolution sub bands LL, LH, HL and HH. LL sub bands represents high scale low frequency coefficient set, sub band LH represents horizontal details of low scale high frequency set and HL sub band represents vertical details of low scale high frequency set and HH sub band is diagonal details low scale high frequency set of an image [8].

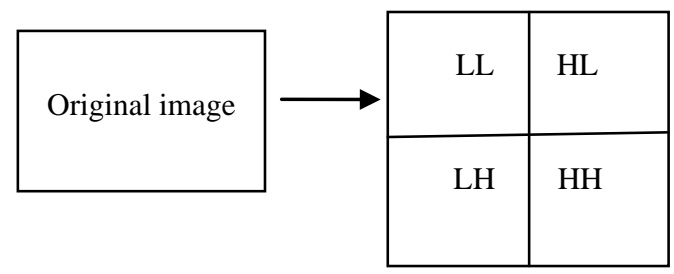

Fig 1: DWT decomposition

Singular value decomposition (SVD) is decomposition of a matrix into three orthogonal matrices of same size that is factorization of matrix. Let assume A is matrix on which SVD is applied then:

$$
\operatorname{SVD}(\mathrm{A})=\mathrm{USV}^{\mathrm{T}}
$$

Where $\mathrm{U}$ is unitary matrix which is called left singular values and $\mathrm{V}$ is also a unitary matrix called right singular values. $\mathrm{S}$ is a diagonal matrix which contain diagonal positive values of original matrix A [7]. The method can be explained in two stages: watermark embedding and watermark extraction.

\subsection{Watermark embedding stage}

The steps to embed watermark are as below:

1) In watermark embedding stage first of all discrete wavelet transform (DWT) is applied on an input host image to decompose the input image into four sub bands LL, LH, HL and HH sub bands.

2) Singular value decomposition (SVD) technique is applied on the selected high frequency HH sub band of the host image.

$\mathrm{I}=\mathrm{U}^{\mathrm{I}} \mathrm{S}^{\mathrm{I}} \mathrm{V}^{\mathrm{I}}$

3) Apply DWT to watermark image and decompose it into sub bands.

4) SVD technique is applied on HH sub band of the watermark image.

$\mathrm{WI}=\mathrm{U}^{\mathrm{WI}} \mathrm{S}^{\mathrm{WI}} \mathrm{V}^{\mathrm{WI}}$

5) Least significant bit (LSB) technique is used embed singular values of watermark image in the host image.

6) Then inverse singular value decomposition is applied on transformed host image.

7) Modified coefficients of high frequency $\mathrm{HH}$ sub band are used to apply inverse DWT results in obtaining watermarked image.
Block diagram of procedure of watermark embedding stage Figure 1.

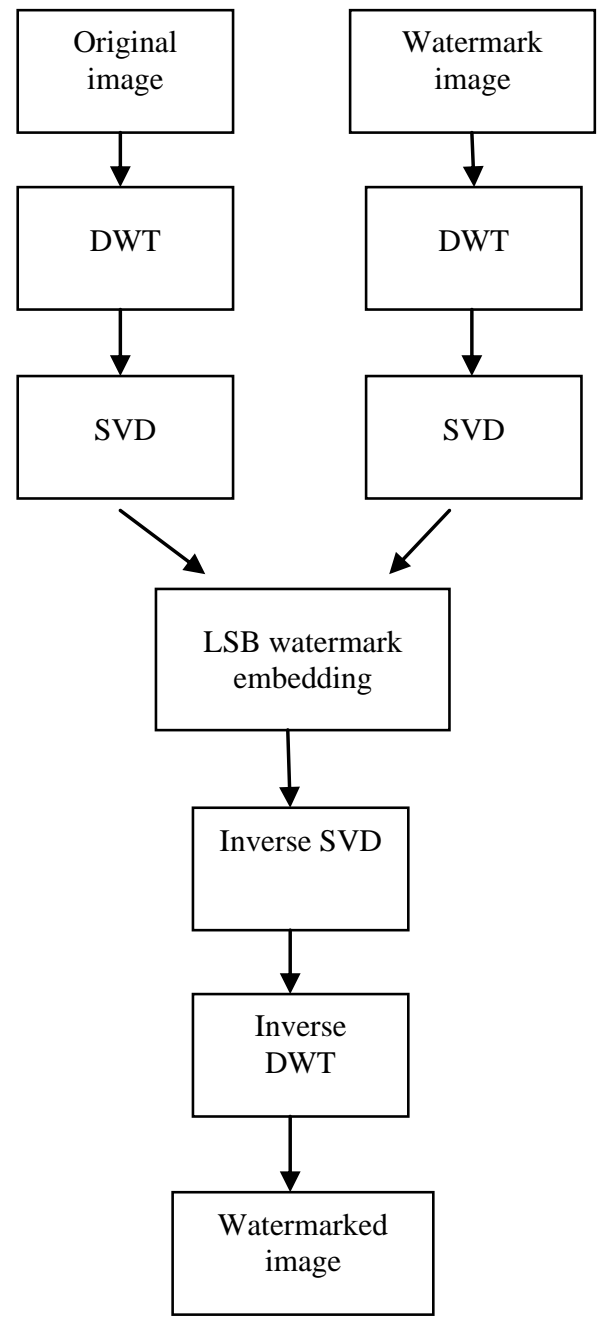

Fig 2: Watermark embedding block diagram

\subsection{Watermark extraction stage}

Steps followed in watermark extraction stage are:

1) In watermark extraction first discrete wavelet transform (DWT) is applied to the watermarked image to divide image into four sub bands.

2) Then singular value decomposition (SVD) is applied to high frequency $\mathrm{HH}$ sub band of watermarked image.

3) After decomposition least significant bit (LSB) technique is applied to extract the embedded watermark image.

4) Then apply inverse SVD technique.

5) Apply inverse discrete wavelet transform (IDWT) to obtain the embedded watermark image. 
Block diagram of watermark extraction stage procedure in Figure 2.

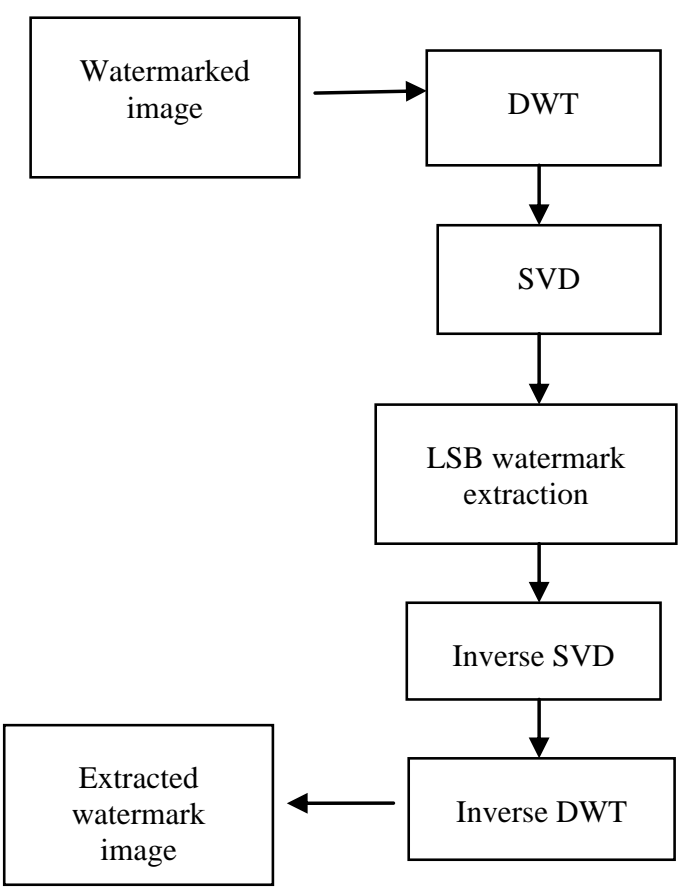

Fig 3: Watermark extraction block diagram

\section{EXPERIMENTAL RESULTS OF PROPOSED TECHNIIQUE}

In this proposed method gray scale Lena image of size $512 * 512$ is used as an original host image and gray scale Baboon image is used as watermark image of size $512 * 512$.
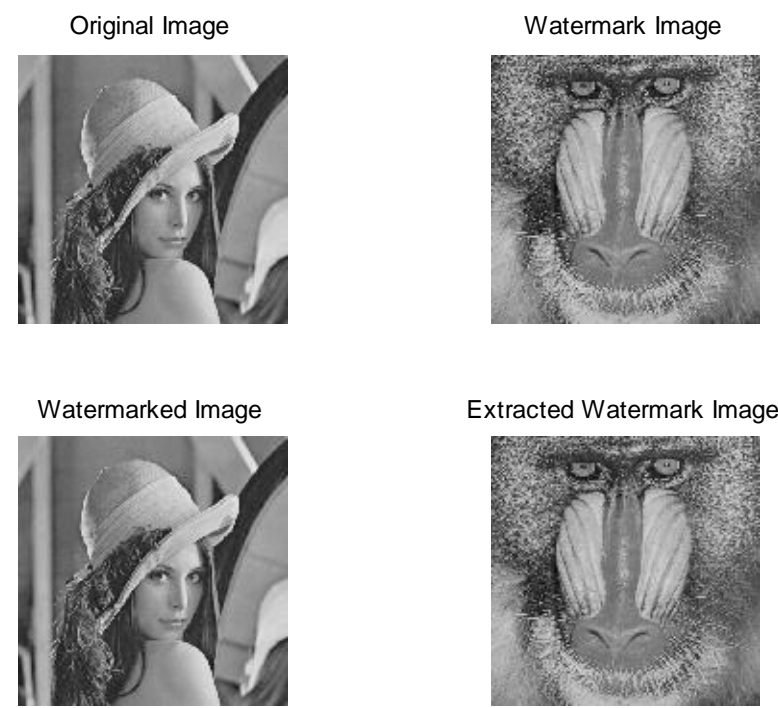

Fig 4: Images used and obtained in proposed method

To check the quality of watermark image some performance measurement metrics are used such as peak signal to noise ratio (PSNR), mean square root (MSE) and structural similarity index measurement (SSIM).Mean Square Error (MSE) can be measured as average of square of errors (difference between watermarked image and original image) [9].

$$
\mathrm{MSE}=\frac{1}{m n} \sum_{i=0}^{m-1} \sum_{j=0}^{n-1}[I(i, j)-K(i, j)]^{2}
$$

Where $\mathrm{m}$ is number of row and $\mathrm{n}$ is number of columns and $\mathrm{I}(i, j)$ is an original image and $\mathrm{K}(i, j)$ is watermarked image.

Peak Signal to Noise Ratio defined as ratio between maximum power of signal and power of corrupting noise. Generally PSNR is defined in terms of MSE which for $m^{*} n$ images $\mathrm{I}$ and $\mathrm{K}$ where one of images is considered as noisy approximation or watermarked version of other [9].

$$
\begin{aligned}
\text { PSNR } & =10 * \log _{10}\left(\frac{M a x^{2}}{M S E}\right) \\
& =20 * \log _{10}\left(\frac{M a x}{\sqrt{M S E}}\right)
\end{aligned}
$$

Max is maximum possible value of pixel of image. If pixels are represented by using 8 bits per pixel then Max is 255 $\left(\mathrm{Max}=2^{8}-1=255\right)$ [8]. Typical values of PSNR are between $30 \mathrm{~dB}$ and $40 \mathrm{~dB}$ for 8 bit data and between $60 \mathrm{~dB}$ and $80 \mathrm{~dB}$ for 16 bit data. PSNR is used to measure amount of visual quality degradation between original image and watermarked image.

Structural similarity index measure is a method for measuring the similarity between two images. The SSIM is a decimal value between -1 and 1 . The value 1 is reached only if there are two identical sets of data [10].

Some attacks (like median filter and addition of noise) are also performed on watermarked image to check the robustness of watermark in Figure 5, Figure 6 and Figure 7.
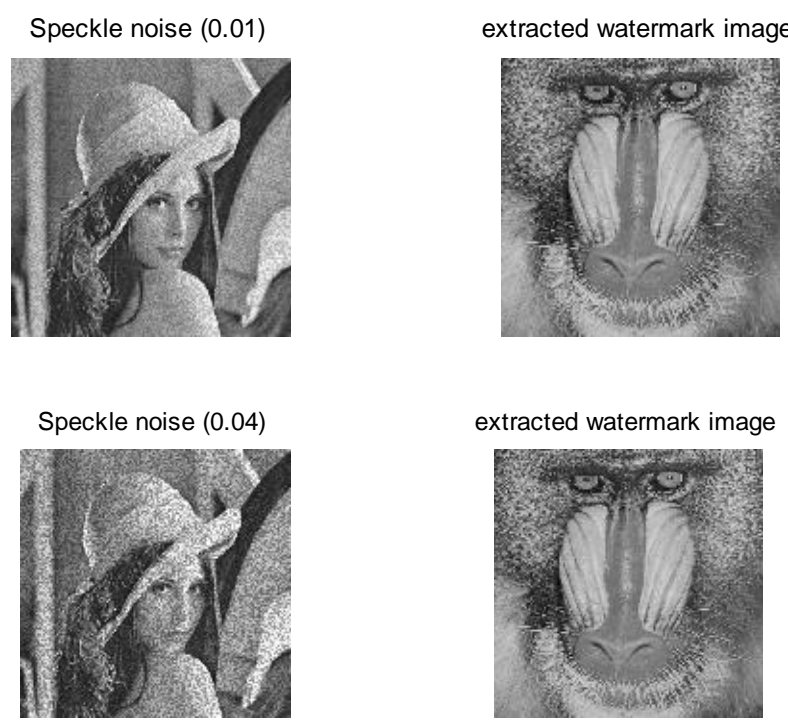

Fig 5: Speckle noise and extracted watermark

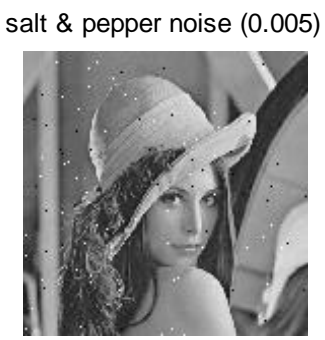

extracted watermark image

Fig 6: Salt and pepper noise attack and extracted watermark 


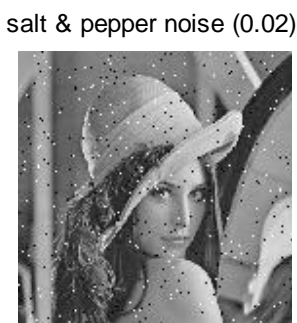

extracted watermark image median filter $\left[3^{\star} 3\right]$

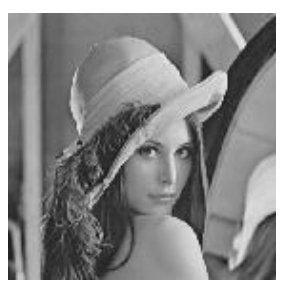

median filter $\left[5^{\star} 5\right]$

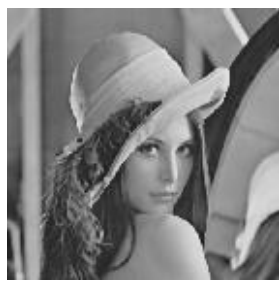

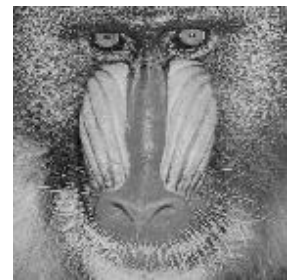

extracted watermark image

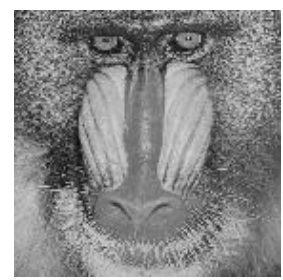

extracted watermark image

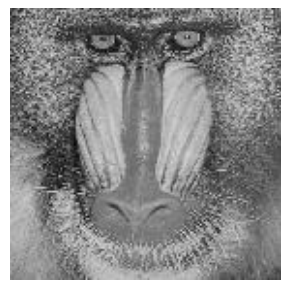

Fig 7: Salt \& pepper noise attack and median filter attacks

Results of comparison between proposed method based on discrete wavelet transform, least significant bit technique and singular value decomposition technique and the watermarking scheme based on redundant discrete wavelet technique and singular value decomposition technique are as in Table 1 , Table 2 and Table 3.

Table 1. Comparison between robust watermarking scheme based on RDWT- SVD and proposed watermarking method (PSNR)

\begin{tabular}{|c|c|c|}
\hline Image/ Attacks & $\begin{array}{c}\text { Robust scheme } \\
\text { based on RDWT } \\
\text { and SVD }\end{array}$ & $\begin{array}{c}\text { Proposed method } \\
\text { based on DWT, } \\
\text { LSB and SVD }\end{array}$ \\
\hline Watermarked image & 37.6758 & 42.3432 \\
\hline Speckle noise (0.01) & 25.1886 & 25.5689 \\
\hline $\begin{array}{c}\text { Salt and Pepper } \\
\text { noise (0.02) }\end{array}$ & 22.2236 & 22.4915 \\
\hline $\begin{array}{c}\text { Median filter [3*3] } \\
\text { Speckle noise (0.04) }\end{array}$ & 33.5902 & 35.1513 \\
\hline $\begin{array}{c}\text { Salt and Pepper } \\
\text { noise (0.005) }\end{array}$ & 28.0104 & 19.7403 \\
\hline Median filter [5*5] & 30.5246 & 31.2263 \\
\hline
\end{tabular}

Table 2. Comparison of MSE between watermarking scheme based on RDWT- SVD and proposed watermarking scheme based on DWT-LSB-SVD

\begin{tabular}{|c|c|c|}
\hline Image/ Attacks & $\begin{array}{c}\text { Robust scheme } \\
\text { based on RDWT } \\
\text { and SVD }\end{array}$ & $\begin{array}{c}\text { Proposed method } \\
\text { based on DWT, } \\
\text { LSB and SVD }\end{array}$ \\
\hline Watermarked image & 11.1044 & 3.7911 \\
\hline $\begin{array}{c}\text { Speckle noise (0.01) } \\
\text { MSE } \\
\text { noise (0.02) }\end{array}$ & 196.8871 & 180.3805 \\
\hline $\begin{array}{c}\text { Median filter [3*3] } \\
\text { Speckle noise (0.04) }\end{array}$ & 721.3031 & 366.3777 \\
\hline $\begin{array}{c}\text { Salt and Pepper } \\
\text { noise (0.005) }\end{array}$ & 102.8106 & 19.8588 \\
\hline Median filter [5*5] & 57.6258 & 49.038 .3268 \\
\hline
\end{tabular}

Table 3. SSIM comparison

\begin{tabular}{|c|c|c|}
\hline Image/ Attacks & $\begin{array}{c}\text { Robust scheme } \\
\text { based on RDWT } \\
\text { and SVD }\end{array}$ & $\begin{array}{c}\text { Proposed method } \\
\text { based on DWT, } \\
\text { LSB and SVD }\end{array}$ \\
\hline Watermarked image & 0.9993 & 1.0000 \\
\hline Speckle noise (0.01) & 0.8033 & 0.8087 \\
\hline $\begin{array}{c}\text { Salt and Pepper } \\
\text { noise (0.02) }\end{array}$ & 0.9070 & 0.7218 \\
\hline $\begin{array}{c}\text { Median filter [3*3] } \\
\text { Speckle noise (0.04) }\end{array}$ & 0.9854 & 0.9851 \\
\hline $\begin{array}{c}\text { Salt and Pepper } \\
\text { noise (0.005) }\end{array}$ & 0.9070 & 0.6081 \\
\hline Median filter [5*5] & 0.9403 & 0.9096 \\
\hline
\end{tabular}

\section{CONCLUSION}

In this paper a new combined digital image watermarking technique is presented and from the results of performance metrics it shows that the proposed method of watermarking is efficient based on the quality of the watermarked image obtained and recovered watermark after different attacks. This proposed method provides better image quality and better watermark extraction than robust digital watermarking scheme based on RDWT-SVD. It will provide security to the digital documents and will help to provide copyrights to original owner of digital contents. Further work can be done to implement this scheme for colored images and to check its robustness against geometric attacks.

\section{ACKNOWLEDGEMENT}

Sincere thanks to the fine people around who helped in completion of this research work. Sincere thanks to supervisor Er. Nirvair Neeru, for giving an opportunity to work on topic of digital watermarking. Her continued support, guidance and vision have helped in this research and it has been a pleasure working with her. Her wisdom clarity of thoughts and support bring the research to this state. 


\section{REFERENCES}

[1] Mohan Durvey, Devshri Satyarthi, "A Review Paper on Digital Watermarking", International Journal of Emerging Trends \& Technology in Computer Science, 2014, Vol. 3(4), pp: 99-105.

[2] Chan - I1 Woo and Seung Dae Lee, "Digital Watermarking for Image Tamper Detection using Block Wise Technique", International Journal of Smart Home, 2013, Volume 7(5), pp: 115-124.

[3] Farooq Huain, "A Survey of Digital Watermarking Techniques for Multimedia Data", MIT International Journal of Electronics and Communication Engineering, 2012, Vol. 2, pp. 37-43.

[4] Hanaa A. Abdullah, Rania A. ghazy, Hany Kasban, Osama S. Faragallah, Abdalhameed A Shaalan, Mohiy M. Hadhoud and Fathi E. Abd El-samie, "Homomorphic imagewatermarking with a singular value decomposition algorithm", Information Processing and Management 50,2014, pp: 909-923.

[5] Saeid Fazli and Masoumeh Moeini, "A Robust Image Watermarking Method Based on DWT, DCT and SVD Using a New Technique for Correction of Main Geometric Attacks", Elsevier Science Direct, 2015, pp: 1-9.
[6] Samira Lagzian, Mohsen Soryani and Mahmood Fathy,"A New Robust Watermarking Scheme Based on RDWT-SVD", International Journal of Intelligent Information Processing, 2011, Vol. 2 (1)

[7] V Santhi and Dr. Arunkumar Thangavelu, "DWT-SVD Combined Full Band Robust Watermarking Technique for Color Images in YUV Color Spaces", International Journal of Computer Theory and Engineering, 2009, Volume 1 (4), pp: 424 - 429.

[8] Asna Furqan and Munish Kumar, "Study and Analysis of Robust DWT-SVD Domain Based Digital Image Watermarking Technique Using MATLAB", IEEE International Conference on Computational Intelligence \& Communication Technology, 2015, pp: 638-644.

[9] Abdullah Bamatraf, Rosziati Ibrahim and Mohd. Najib Mohd. Salleh, "A new digital watermarking algorithm using combination of least significant bit (LSB) and inverse bit", Journal of Computing, 2011, Volume 3 (4), pp: $1-8$.

[10] Arathi Chitla and Chandra Mohan M, "Authenticating Medical Images with Lossless Digital Watermarking", International Journal of Multidisciplinary and Current Research, 2014, Volume 2, pp: 291-296 